\title{
PKA and CaMKII mediate PI3K activation in bovine sperm by inhibition of the PKC/PP1 cascade
}

\author{
H Rotfeld, P Hillman, D Ickowicz and H Breitbart \\ The Mina and Everard Faculty of Life Sciences, Bar-Ilan University, Ramat-Gan 5290002, Israel \\ Correspondence should be addressed to H Breitbart; Email: Haim.breitbart@biu.ac.il
}

\begin{abstract}
To enable fertilization, spermatozoa must undergo several biochemical processes in the female reproductive tract, collectively called capacitation. These processes involve protein kinase A (PKA)-dependent protein tyrosine phosphorylation including phosphatidylinositol-3-kinase (PI3K). It is not known how PKA, a serine/threonine (S/T) kinase, mediates tyrosine phosphorylation of proteins. We recently showed that inhibition of S/T phosphatase 1 (PP1) causes a significant increase in phospho-PI3K. In this study, we propose a mechanism by which PKA and PP1 mediate an increase in PI3K tyrosine phosphorylation and implicate calmodulin-dependent kinase II (CaMKII) in this process. Inhibition of sperm PP1 or PKC, stimulated CaMKII phosphorylation/activation, and inhibition of PKC enhanced PP1 phosphorylation/inactivation. Inhibition of CaMKII, using KN-93, caused significant reduction in phospho-PP1, indicating its activation. Moreover, KN-93 prevented the dephosphorylation/inactivation of PKC. We therefore suggest that CaMKII inhibits PKC, leading to PP1 inhibition and the reciprocal auto-activation of CaMKII. Thus, CaMKII can regulate its own activation by inhibiting the PKC/PP1 cascade. Inhibition of Src family kinases (SFK) caused significant inhibition of CaMKII and PP1 phosphorylation, suggesting that SFK activity results in PP1 inhibition and CaMKII activation. Activation of sperm PKA by 8Br-cAMP revealed an increase in phosphoCaMKII, which was inhibited by PKA inhibitor. Tyrosine phosphorylation of PI3K was stimulated by 8Br-cAMP and by PKC or PP1 inhibition and was abrogated by CaMKII inhibition. Furthermore, phosphorylation/activation of the tyrosine kinase Pyk2 was enhanced by PP1 inhibition, and this activation is blocked by CaMKII inhibition. Thus, PKA activates Src, which inhibits PP1, leading to CaMKII and Pyk2 activation, resulting in PI3K tyrosine phosphorylation/activation.

Reproduction (2014) 147 347-356
\end{abstract}

\section{Introduction}

To acquire the ability to fertilize the egg, mammalian spermatozoa must undergo a series of biochemical processes in the female reproductive tract, collectively called capacitation. These processes include cholesterol efflux from the sperm plasma membrane, increased influx of bicarbonate, and $\mathrm{Ca}^{2+}$, which activate soluble adenylyl cyclase (sAC) to produce cAMP, leading to protein kinase $A$ (PKA) activation, protein tyrosine phosphorylation, actin polymerization, and the development of hyperactivated motility (rev. in Breitbart (2003)). The mechanism by which PKA, a serine/threonine (S/T) kinase, mediates protein tyrosine phosphorylation is not fully understood. In murine sperm, PKA co-immunoprecipitates with the tyrosine kinase $\mathrm{Src}$, resulting in Src phosphorylation (Baker et al. 2006). We recently reported that PKA can activate Src in bovine sperm (Etkovitz et al. 2009).

We have previously shown that PKA activation leads to PKC inhibition (Cohen et al. 2004) and that PKA mediates phosphatidylinositol-3-kinase (PI3K) phosphorylation on Tyr-458 and actin polymerization in bovine sperm
(Etkovitz et al. 2007). PKA can directly phosphorylate the PI3K (p85) regulatory subunit on Ser-83, leading to its activation (Cosentino et al. 2007, De Gregorio et al. 2007). PI3K $\alpha$ belongs to PI3K class $1 \mathrm{~A}$ enzymes, which are primarily responsible for the production of $\mathrm{PIP}_{3(3,4,5)}$ in response to growth factors (Cantley 2002). PI3K is implicated in many biological processes, including cell survival, growth, movement and adhesion, protein synthesis, and cytoskeletal rearrangement. A role for $\mathrm{PI} 3 \mathrm{~K}$ has been suggested in sperm functions during capacitation and in the acrosome reaction (Fisher et al. 1998, Etkovitz et al. 2007, Jungnickel et al. 2007). The $\mathrm{PI} 3 \mathrm{~K}$ catalytic and regulatory subunits are present in sperm (Jungnickel et al. 2007), and the PI3K catalytic subunit inhibitor wortmannin $(10 \mathrm{nM})$ inhibits PI3K and $\mathrm{PIP}_{3}$ production in bovine sperm (Etkovitz et al. 2007). In other cell types, it has been shown that PKC $\alpha$ can inhibit PI3K activity directly (Sipeki et al. 2006) or indirectly (Guan et al. 2007). In bovine sperm, activation of PKC leads to PI3K inhibition (Etkovitz et al. 2007).

The PKC $\alpha$ isoform belongs to the classical PKC subfamily that comprises $\mathrm{S} / \mathrm{T}$ kinases and can regulate 
cell proliferation, differentiation, and apoptosis by affecting ion channels, receptors, and enzymes (rev. by Hofmann (2004)). In spermatozoa, PKC was found to participate in sperm capacitation, acrosome reaction, and motility (Breitbart et al. 1992, Breitbart \& Naor 1999, Cohen et al. 2004, Almog et al. 2008).

A direct role for PKC $\alpha$ in the activation of the $S / T$ phosphatase PP2 resulting in PI3K inhibition through removal of PI3K phosphate was shown in epithelial cells (Guan et al. 2007). Both the S/T phosphatases PP1 and PP2 are present in sperm and are found to participate in sperm capacitation, acrosome reaction, and motility (Furuya et al. 1993, Ashizawa et al. 2006, Chakrabarti et al. 2007). In mammals, four PP1c isoforms were identified: PP1 $\alpha, P P 1 \beta, P P 1 \gamma 1$, and PP1 $\gamma 2$, in which $P P 1 \gamma 2$ is testis/sperm specific and important for sperm motility and morphological processes during spermatogenesis (Chakrabarti et al. 2007).

Very little is known about the role and mechanisms of action of PP1 in spermatozoa. In a recent study, we proposed a signaling pathway that involves the regulation of PI3K activation by $\mathrm{PKC} \alpha, \mathrm{PKA}$, and PP1 $\gamma 2$ in bovine sperm capacitation (Rotman et al. 2010). The $\mathrm{Ca}^{2+}$ /calmodulin-dependent kinase II (CaMKII) is a widely distributed $\mathrm{S} / \mathrm{T}$ kinase that mediates flagellar and ciliary motility (Smith 2002, Mwimbi et al. 2003, Nomura et al. 2004). Several studies have implicated CaMKII in sperm capacitation (Ackermann et al. 2009). It was also shown that CaMKII mediates the development of hyper-activated motility, a known parameter of sperm capacitation (Ignotz \& Suarez 2005) and regulates protein tyrosine phosphorylation (González-Fernández et al. 2013), two known parameters of sperm capacitation.

In this study, we propose CaMKII as a key enzyme in the regulation of PI3K activation, which activates Pyk2 to phosphorylate $\mathrm{PI} 3 \mathrm{~K}$ on Tyr-458. We suggest that CaMKII can undergo self-regulation by inhibiting PKC leading to inhibition of PP1, resulting in CaMK activation. Another pathway to activate CaMK involves PKA-dependent activation of Src, which in turn inhibits PP1 leading to CaMKII activation.

\section{Materials and methods}

\section{Materials and antibodies}

Calyculin A, bisindolylmaleimide I (GF109203X (GF)), KN-93, and SU6656 were purchased from Calbiochem (San Diego, CA, USA). Rabbit polyclonal anti-PKC $\alpha$, rabbit polyclonal anti-phospho-PKC $\alpha$ (Thr-497), and polyclonal anti- $\beta$-actin (C4)-HRP were purchased from Santa Cruz Biotechnology. Rabbit polyclonal anti-phospho-PP1 $\alpha$ (Thr-320), rabbit polyclonal antiphospho-PI3K (Tyr-458), and rabbit polyclonal antiphospho-Pyk2 (Tyr-402) were purchased from Cell Signaling (Beverly, MA, USA). Rabbit polyclonal antiphospho-CaMKIl $\alpha$ (Thr-286) was purchased from Abcam
(Cambridge, UK). Mouse monoclonal anti-CaMKIl $\alpha$ was purchased from Millipore (Billerica, MA, USA). Rabbit-anti-PP1 $\gamma 2$ was kindly provided by Prof. S Vijayaraghavan (Department of Biological Sciences, Kent State University, Kent, $\mathrm{OH}$, USA). All other chemicals were purchased from Sigma-Aldrich Israel Ltd., unless otherwise stated.

\section{Sperm preparation}

Ejaculated bull spermatozoa were obtained using artificial vagina, and the 'swim-up' technique was applied to obtain motile sperm. Bovine sperm was supplied by the SION Artificial Insemination Center (Hafetz-Haim, Israel). Sperm cells were washed three times by centrifugation $\left(780 \mathrm{~g}\right.$ for $10 \mathrm{~min}$ at $25^{\circ} \mathrm{C}$ ) in NKM buffer $(110 \mathrm{mM} \mathrm{NaCl}, 5 \mathrm{mM} \mathrm{KCl}$, and $20 \mathrm{mM} \mathrm{3-N-}$ morpholino propane sulfonic acid (MOPS) (pH 7.4)) and the sperm were allowed to swim up after the last wash. The washed cells were counted and maintained at $39{ }^{\circ} \mathrm{C}$ until use. Only sperm samples that contained at least $80 \%$ motile sperm were used in the experiments.

\section{Sperm capacitation}

In vitro capacitation of bovine sperm was induced as described previously (Parrish et al. 1999). Briefly, sperm pellets were re-suspended to a final concentration of $10^{8}$ cell $\mathrm{s} / \mathrm{ml}$ in mTALP (modified Tyrode solution) medium $\left(100 \mathrm{mM} \mathrm{NaCl}, 3.1 \mathrm{mM} \mathrm{KCl}, 1.5 \mathrm{mM} \mathrm{MgCl}_{2}, 0.92 \mathrm{mM}\right.$ $\mathrm{KH}_{2} \mathrm{PO}_{4}, 25 \mathrm{mM} \mathrm{NaHCO}, 20 \mathrm{mM}$ HEPES (pH 7.4), $0.1 \mathrm{mM}$ sodium pyruvate, $21.6 \mathrm{mM}$ sodium lactate, $10 \mathrm{IU} / \mathrm{ml}$ penicillin, $1 \mathrm{mg} / \mathrm{ml}$ BSA, $20 \mu \mathrm{g} / \mathrm{ml}$ heparin, and $2 \mathrm{mM} \mathrm{(aCl}$ ). The cells were incubated in this capacitation medium for $4 \mathrm{~h}$ at $39^{\circ} \mathrm{C}$. The capacitation state of the sperm was routinely confirmed after the 4-h incubation in mTALP by examining the ability of the sperm to undergo the acrosome reaction induced by the addition of the calcium ionophore A23187 $(10 \mu \mathrm{M})$.

\section{Assessment of sperm acrosome reaction}

Washed cells $\left(10^{8}\right.$ cells $\left./ \mathrm{ml}\right)$ were capacitated for $4 \mathrm{~h}$ at $39^{\circ} \mathrm{C}$ in mTALP medium. Acrosome reaction (AR) inducer was then added for another 20 min of incubation. The percentage of acrosome-reacted sperm was determined microscopically using FITC-conjugated Pisum sativum agglutinin (PSA). An aliquot of spermatozoa $\left(10^{6}\right.$ cells) was smeared on a glass slide and allowed to air-dry. The sperm were then permeabilized with methanol for $15 \mathrm{~min}$ at room temperature, washed three times at 5-min intervals with TBS, air dried, and then incubated with FITC-PSA ( $50 \mu \mathrm{g} / \mathrm{ml}$ in TBS) for $30 \mathrm{~min}$, washed twice with $\mathrm{H}_{2} \mathrm{O}$ at 5 -min intervals, and mounted with FluoroGuard Antifade (Bio-Rad Laboratories). For each experiment, at least 200 cells/slide on duplicate 
slides were evaluated (total of 400 cells for one experiment). Cells with green staining over the acrosomal cap were considered acrosome intact; those with equatorial green staining or no staining were considered acrosome reacted.

\section{Immunoblot analysis}

Sperm were washed by centrifugation for $5 \mathrm{~min}$ at $10000 \mathrm{~g}$ at $4{ }^{\circ} \mathrm{C}$ and then the supernatant was discarded and the pellet was re-suspended in TBS and centrifuged again to remove remaining traces of BSA. Sperm lysates were prepared by the addition of lysis buffer $(50 \mathrm{mM}$ Tris- $\mathrm{HCl}, \mathrm{pH} 7.5,150 \mathrm{mM} \mathrm{NaCl}, 6 \%$ SDS, protease inhibitor cocktail 1:100, $50 \mu \mathrm{M} \mathrm{NaF}, 50 \mu \mathrm{M}$ Na-pyrophosphate, $0.2 \mathrm{mM} \mathrm{Na} V_{3} \mathrm{O}_{4}$, and freshly added $1 \mathrm{mM}$ phenylmethylsulfonyl fluoride), to the pellet, and the lysate vortexed vigorously for $15 \mathrm{~min}$ at room temperature. Lysates were then centrifuged for $5 \mathrm{~min}$ at $10000 \mathrm{~g}$ at $4{ }^{\circ} \mathrm{C}$, the supernatant was transferred, and the protein concentration was determined by the bicinchoninic acid (BCA) method (Smith et al. 1985). Sample buffer $5 \times$ was added to the supernatant, and the sample was boiled for $5 \mathrm{~min}$. The extracts were separated on 7 or $10 \%$ SDS-polyacrylamide gels and then electrophoretically transferred to nitrocellulose membranes. Blots were routinely stained with Ponceau solution to confirm equal loading and even transfer. The blots were blocked with $1 \%$ BSA in Tris-buffered saline, $\mathrm{pH}$ 7.6, containing $0.1 \%$ Tween 20 (TBST), for $30 \mathrm{~min}$ at room temperature. The membranes were incubated overnight at $4{ }^{\circ} \mathrm{C}$ with the primary antibodies diluted in $1 \%$ BSA in TBST. Next, the membranes were washed three times with TBST and incubated for $1 \mathrm{~h}$ at room temperature with specific HRP-linked secondary antibodies (Bio-Rad Laboratories), diluted 1:5000 in TBST and $1 \%$ BSA. The membranes were washed three times with TBST and visualized by ECL (Amersham).

\section{Results}

We previously showed an increase in tyrosine phosphorylation of $\mathrm{PI} 3 \mathrm{~K}$ during capacitation of bovine sperm (Etkovitz et al. 2007). We also showed that the level of this phosphorylation is significantly enhanced by treating the sperm cells with calyculin A, a S/T phosphatase inhibitor with high specificity toward PP1 (Rotman et al. 2010). In this study, we explain the mechanism by which the inhibition of the S/T phosphatase PP1 enhances the tyrosine phosphorylation level of PI3K.

\section{CaMKII phosphorylation is affected by PP1, PKC, and SrC}

Sperm incubated under capacitation conditions in the presence of the PP1 inhibitor, calyculin A, showed

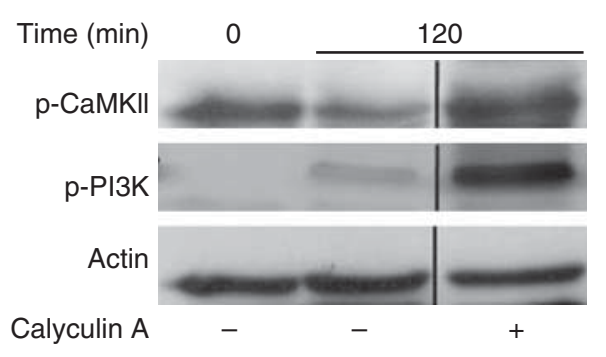

Figure 1 PP1 inactivation enhances CaMKII and PI3K phosphorylation. Bovine spermatozoa were incubated in capacitation medium with or without calyculin A (100 nM) for $2 \mathrm{~h}$. Proteins were extracted and analyzed by western blot using anti-phospho-PI3K, anti-phosphoCaMKII $\alpha$, and anti-actin (loading control) antibodies. The results shown are representative of three independent experiments.

significant enhancement of PI3K phosphorylation on tyrosine 458 and CaMKII on threonine 286 (Fig. 1). As inhibition of PP1 also enhanced CaMKII phosphorylation (Fig. 1) and it was previously shown that CaMKII can activate the tyrosine kinase Pyk2 (Della Rocca et al. 1997, Soltoff 1998, Zwick et al. 1999, Heidinger et al. 2002, Fan et al. 2005, Montiel et al. 2007), which can tyrosine phosphorylate PI3K (Dikic et al. 1996, Schlaepfer \& Hunter 1996, Avraham et al. 2000, Rocic et al. 2001), we also tested whether CaMKII can mediate PI3K tyrosine phosphorylation in sperm capacitation. CaMKII was already phosphorylated at the beginning of the capacitation process, with some decrease in the phosphorylation after $2 \mathrm{~h}$ of incubation (Fig. 2). The calmodulin inhibitor $\mathrm{W}-7$ caused significant reduction in p-CaMKII (Fig. 2). It is known that CaMKII undergoes self-phosphorylation, and inhibition of this activity by $\mathrm{KN}-93$ causes significant reduction in $\mathrm{p}-\mathrm{CaMK}$ (as shown later), indicating that $\mathrm{KN}-93$ can be used as CaMKII inhibitor in sperm cells. $\mathrm{KN}-93$ is a membranepermeable CaMKII inhibitor that prevents $\mathrm{Ca}^{2+}$-calmodulin binding to CaMKII and thereby inhibits its activation (Hudmon \& Schulman 2002, Hunter \& Schulman 2005). No change in percent of motile sperm was observed in KN-93-treated sperm.

It is known that PP1 dephosphorylates/inactivates CaMKII in other cell types (Hwang et al. 1996, Lisman \& Zhabotinsky 2001). It was shown elsewhere (Srinivasan \& Begum 1994) that activation of PKC leads to PP1 activation. We suggested that PKC $\alpha$ mediates the activation of PP1 in bovine sperm (Rotman et al. 2010). Thus, we assumed that inhibition of PKC would affect CaMKII activity via inhibition of PP1. To detect the phosphorylation state of PP1, we used an antibody against $\mathrm{p}$-(Thr-320)-PP1 $\alpha$ able to recognize phosphorylated PP1 $\gamma 2$. It has been shown elsewhere (Huang \& Vijayaraghavan 2004) that an antibody against p-PP1 $\alpha$ can recognize phosphorylated PP1 $\gamma 2$. This is due to the fact that testis- and sperm-specific PP1 $\gamma 2$ has the sequence of TRPTPPR, which differs by only one amino acid from the corresponding sequence in PP1 $\alpha$ but is the 


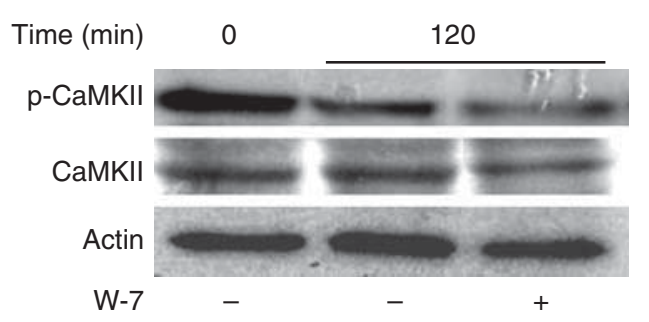

Figure 2 Phosphorylation of CaMKII in sperm capacitation. Bovine spermatozoa were incubated in MTALP (capacitation medium) with or without the CaM inhibitor W-7 $(50 \mu \mathrm{M})$ for $2 \mathrm{~h}$. Proteins were extracted at the indicated times and analyzed by western blot using antiphospho-CaMKIl $\alpha$, anti-CaMKIl $\alpha$, and anti-actin (loading control) antibodies. The results shown are representative of three independent experiments.

same as that of PP1 $\gamma 1$. Sperm treatment with GF, a PKC inhibitor, caused an increase in p-PP1 and p-CaMKII, indicating PP1 inactivation and CaMKII activation (Fig. 3A). Thus, as was found for direct inhibition of PP1 by calyculin A (Fig. 1), indirect inhibition of PP1 by inhibiting PKC activates CaMKII. It was suggested elsewhere that the Src family kinases (SFK) are able to phosphorylate PP1 $\alpha$ resulting in PP1 inactivation (Srinivasan \& Begum 1994, Villa-Moruzzi \& Puntoni 1996, Mallozzi et al. 2005, Mao et al. 2005). We assumed that sperm PP1 might also be inhibited by SFK, and indeed, we showed that treatment of sperm with SU6656, a known SFK inhibitor, caused a decrease in CaMKII and PP1 phosphorylation levels (Fig. 3B). These data suggest that dephosphorylation/activation of PP1 by inhibiting SFK causes inactivation of CaMKII. Moreover, we found that the CaMKII inhibitor KN-93 caused a dephosphorylation/activation of p-PP1 (Fig. 3C) and prevented the dephosphorylation/inactivation of PKC $\alpha$ (Fig. 3C), suggesting that CaMK inhibits PP1 activation through inactivation of $\mathrm{PKC} \alpha$.

\section{PKA mediates CaMKII phosphorylation}

In our previous study, we showed that activation of sperm PKA causes significant increase in tyrosine phosphorylation of PI3K (Etkovitz et al. 2007) and further suggested that PKA can activate Src, leading to EGFR phosphorylation on Tyr-845, the site known to be phosphorylated by Src (Etkovitz et al. 2009). It has been proposed that Src is activated by a cAMP-mediated pathway via inhibition of Csk, the tyrosine kinase responsible for inactivation of $\mathrm{c}-\mathrm{Src}$ in other cell types (Baker et al. 2006). Here, we showed that inhibition of Src by SU6656 caused a significant reduction in phospho-CaMKII (Fig. 3B). Thus, we assumed that PKA might mediate CaMKII phosphorylation. The data in Fig. 4A show that $\mathrm{H}-89$, a known PKA inhibitor, caused significant reduction in $\mathrm{p}$-CaMKII, and addition of the permeable $8 \mathrm{Br}$-cAMP, which activates PKA, enhanced p-CaMKII (Fig. 4B). Addition of 8Br-cAMP enhanced PI3K phosphorylation, which was inhibited by the
CaMKII inhibitor KN-93 (Fig. 4C). Thus, a possible mechanism by which PKA mediates CaMKII phosphorylation is as follows: PKA activates Src which in turn blocks PP1 leading to an increase in p-CaMKII.
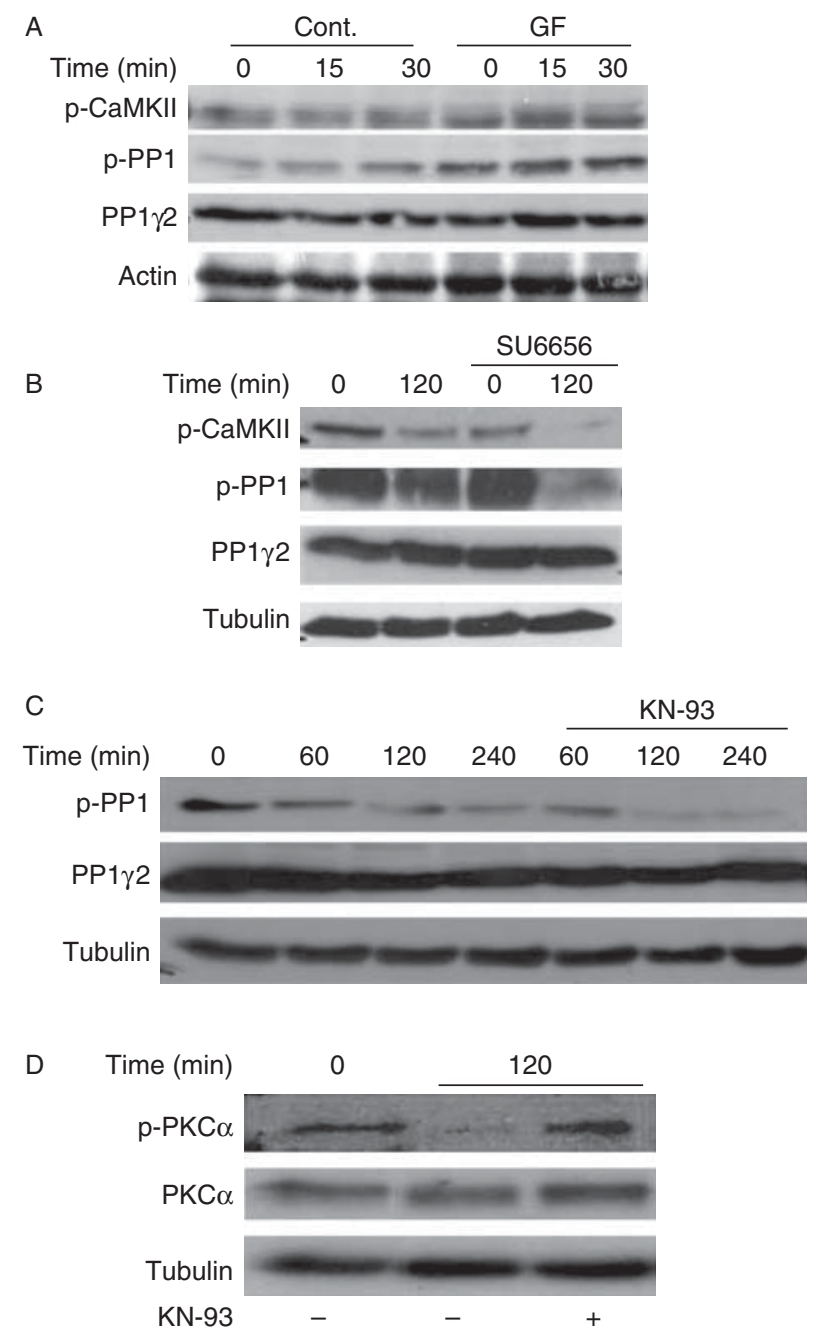

Figure 3 The dual relationship between PKC/PP1 and CaMKII. (A) Bovine spermatozoa were incubated in NKM (starvation medium) for $1.5 \mathrm{~h}$ with or without GF109203X (0.1 nM) and then transferred to mTALP (capacitation medium) for 15 or $30 \mathrm{~min}$. Proteins were extracted and analyzed by western blot using anti-PP1 $\gamma 2$, anti-phosphor-PP1 $\alpha$, anti-phospho-CaMKII, and anti-actin (loading control) antibodies. (B) Bovine spermatozoa were incubated in NKM (starvation medium) for $1.5 \mathrm{~h}$ with or without SU6656 $(50 \mu \mathrm{M})$ and then transferred to mTALP (capacitation medium) for $2 \mathrm{~h}$. Proteins were extracted and analyzed by western blot using anti-PP1 $\gamma 2$, anti-phospho-PP1, anti-phospho-CaMKII, and anti-tubulin (loading control) antibodies. (C) Bovine spermatozoa were incubated in capacitation medium with or without KN-93 $(50 \mu \mathrm{M})$ for $4 \mathrm{~h}$. Proteins were extracted at the indicated times and analyzed by western blot using anti-phospho-PP1 $\alpha$, anti-PP1 $\gamma 2$, and anti-tubulin (loading control) antibodies. (D) Bovine spermatozoa were incubated in capacitation medium with or without KN-93 $(50 \mu \mathrm{M})$ for $2 \mathrm{~h}$. Proteins were extracted and analyzed by western blot using anti-phosphoPKC $\alpha$, anti-PKC $\alpha$, and anti-tubulin (loading control) antibodies. The results shown are representative of three independent experiments. 
A

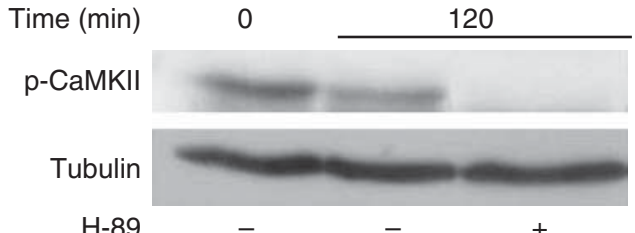

B

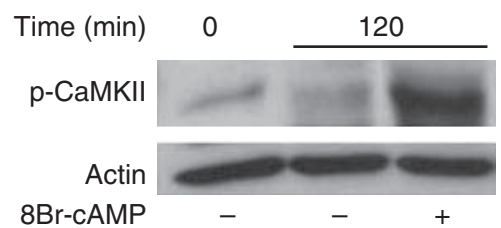

C Time $(\min ) \quad 0$

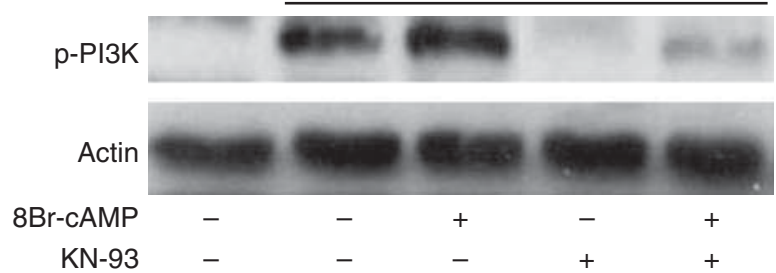

Figure 4 The effect of PKA activation on CaMKII. (A) Bovine spermatozoa were incubated in capacitation medium with or without $\mathrm{H}-89(50 \mu \mathrm{M})$ for $2 \mathrm{~h}$. Proteins were extracted and analyzed by western blot using anti-phospho-CaMKII and anti-tubulin (loading control) antibodies. (B) Bovine spermatozoa were incubated in capacitation medium with or without $8 \mathrm{Br}$-cAMP $(1 \mathrm{mM})$ for $2 \mathrm{~h}$. Proteins were extracted and analyzed by western blot using anti-phospho-CaMKII and anti-actin (loading control) antibodies. (C) Bovine spermatozoa were incubated in capacitation medium with or without $\mathrm{KN}-93$ $(50 \mu \mathrm{M})$ or $8 \mathrm{Br}-\mathrm{cAMP}(1 \mathrm{mM})$ for $2 \mathrm{~h}$. Proteins were extracted and analyzed by western blot using anti-phospho-PI3K and anti-actin (loading control) antibodies. The results shown are representative of three independent experiments.

\section{Tyrosine phosphorylation of PI3K is affected by CaMKII, PKC, and PP1}

Sperm incubated under capacitation conditions showed an increase in tyrosine phosphorylation/activation of $\mathrm{PI} 3 \mathrm{~K}$ after $2-4 \mathrm{~h}$ of incubation (Fig. 5A). This phosphorylation was abrogated in cells treated with $\mathrm{KN}-93$ (Fig. 5A), indicating the involvement of CaMKII in PI3K activation. Inhibition of PKC using GF or long incubation with 12-o-tetradecanoyl phorbol-13-acetate (PMA) revealed a significant increase in $\mathrm{p}-\mathrm{PI} 3 \mathrm{~K}$, which was completely blocked by KN-93 (Fig. 5B). Moreover, inhibition of PP1 by calyculin A also enhanced p-PI3K, and the increased phosphorylation was completely blocked by $\mathrm{KN}-93$ (Fig. 5C). These results suggest that CaMK might inhibit PKC, leading to inhibition of PP1, and resulting in an increase of p-PI3K. Indeed, as shown in Fig. 3D, KN-93 prevented the dephosphorylation/ inactivation of PKC during sperm capacitation, indicating that CaMKII induced inhibition of PKC activity.

\section{CaMKII activates the tyrosine kinase PyK2}

In other cell types, CaMKII can activate Pyk2 (Della Rocca et al. 1997, Soltoff 1998, Zwick et al. 1999, Heidinger et al. 2002, Fan et al. 2005, Montiel et al. 2007), which can activate PI3K (Dikic et al. 1996, Schlaepfer \& Hunter 1996, Avraham et al. 2000, Rocic et al. 2001). Thus, we expected Pyk2 to be activated by CaMKII during sperm capacitation, leading to PI3K activation. Indeed, we found that calyculin A enhanced Pyk2 phosphorylation on tyrosine 402, and this phosphorylation was blocked by KN-93 (Fig. 6). These data indicate that CaMKII activates Pyk2. Moreover, the enhanced phosphorylation by calyculin A of CaMKII on threonine and of Pyk2 on tyrosine indicates that PP1 indirectly affects the Pyk2 phosphorylation state and

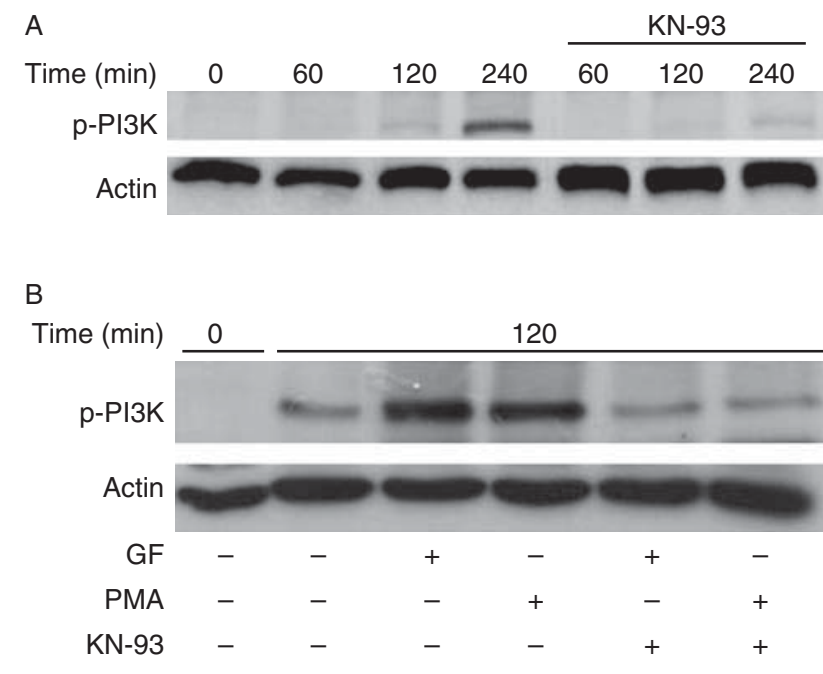

C

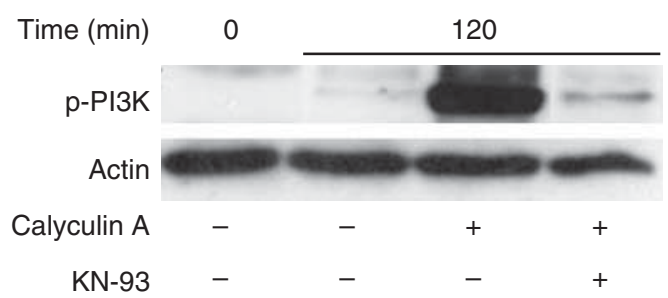

Figure 5 PP1 inactivation enhances PI3K phosphorylation through activation of CaMKII. (A) Bovine spermatozoa were incubated in capacitation medium with or without KN-93 $(50 \mu \mathrm{M})$ for 4 h. Proteins were extracted at the indicated times and analyzed by western blot using anti-phospho-PI3K and anti-actin (loading control) antibodies. (B) Bovine spermatozoa were incubated in capacitation medium with or without KN-93 $(50 \mu \mathrm{M})$ for $10 \mathrm{~min}$ and then PMA $(100 \mathrm{ng} / \mathrm{ml})$ or GF109203X (0.1 nM) was added for additional $2 \mathrm{~h}$ of incubation. Proteins were extracted and analyzed by western blot using antiphospho-PI3K and anti-actin (loading control) antibodies. (C) Bovine spermatozoa were incubated in capacitation medium with or without $\mathrm{KN}-93(50 \mu \mathrm{M})$ for $10 \mathrm{~min}$ and then calyculin $\mathrm{A}(100 \mathrm{nM})$ was added for additional $2 \mathrm{~h}$ of incubation. Proteins were extracted and analyzed by western blot using anti-phospho-PI3K and anti-actin (loading control) antibodies. The results shown are representative of three independent experiments. 


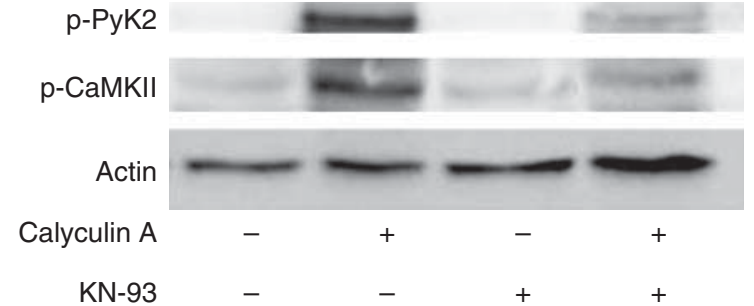

Figure 6 CaMKII activates PyK2 during capacitation. Bovine spermatozoa were incubated in capacitation medium with or without KN-93 $(50 \mu \mathrm{M})$ for $10 \mathrm{~min}$ and then calyculin $\mathrm{A}(100 \mathrm{nM})$ was added for additional $2 \mathrm{~h}$ of incubation. Proteins were extracted and analyzed by western blot using anti-phospho-PyK2, anti-phospho-CaMKII, and antiactin (loading control) antibodies. The results shown are representative of three independent experiments.

further suggests that PP1 dephosphorylates/inactivates CaMKII, leading to dephosphorylation/inactivation of Pyk2.

\section{CaMKII prevents the spontaneous acrosome reaction}

It was shown elsewhere that CaMKII recruits synaptic vesicles to the active zone of the presynaptic nerve terminal (Greengard et al. 1993, Leal-Ortiz et al. 2008). This pathway resembles the situation in the acrosome reaction, in which outer acrosomal membrane-specific sites interact with the overlying plasma membrane. Moreover, it was shown that inhibition of CaMKII using the permeable peptide AIPII or the calmodulin inhibitor $\mathrm{W}-7$, but not $\mathrm{KN}-93$, causes a significant increase in the occurrence of spontaneous acrosomal exocytosis in mouse sperm (Ackermann et al. 2009). We found that incubation of bovine sperm under capacitation conditions in the presence of $\mathrm{KN}-93$ or $\mathrm{W}-7$ caused a significant increase ( 72 and $45 \%$ respectively) in the spontaneous acrosome reaction rate compared with the control cells (24\%) (Fig. 7). Moreover, it was shown that $\mathrm{KN}-93$ inhibits hyper-activated motility in bovine sperm (Ignotz \& Suarez 2005). Thus, it seems that sperm sensitivity to $\mathrm{KN}-93$ differs among species. As the spontaneous acrosome reaction is very high $(72 \%)$ in $\mathrm{KN}$-93-treated sperm, the ionophore-induced acrosome reaction is only $6 \%$ compared with the $48 \%$ found in the control cells. Surprisingly, the ionophore-induced acrosome reaction was inhibited by W-7 (Fig. 7), suggesting that calmodulin might be a positive regulator of the induced acrosome reaction. In conclusion, these data suggest that active CaMKII protects sperm from undergoing spontaneous acrosome reaction, a process known to negatively affect the fertilization rate (Wiser et al. 2013).

\section{Discussion}

In this study, we describe possible mechanisms by which PP1 regulates tyrosine phosphorylation of PI3K. We showed that inhibition of PP1 enhanced PI3K as well as
CaMKII phosphorylation (Fig. 1). In our previous study in bovine sperm, we suggested that PP1 can be activated by PKC (Rotman et al. 2010). Here, inhibition of PKC caused significant increase in $\mathrm{p}$-CaMKII and p-PP1 (Fig. 3A), indicating the activation of CaMKII and inhibition of PP1. Moreover, we show that KN-93 inhibits dephosphorylation/inactivation of $P K C \alpha$ (Fig. 3D), suggesting that CaMKII inhibits PKC $\alpha$ activity by enhancing its dephosphorylation. Altogether, our data suggest that CaMKII can inhibit PKC leading to inactivation of PP1 and in return an increase in CaMKII phosphorylation/activation occurs. Thus, CaMKII can regulate its own activity by the inhibition of $\mathrm{PP} 1 \alpha$ and preventing self-dephosphorylation. This conclusion is supported by reports demonstrating that PP1 can dephosphorylate/inactivate CaMKII (Hwang et al. 1996, Lisman \& Zhabotinsky 2001). Another factor that can affect PP1 is Src (Villa-Moruzzi \& Puntoni 1996, Huang \& Vijayaraghavan 2004), whose inhibition revealed complete reduction/inactivation of $\mathrm{p}$-CaMKII, and reduction/activation of p-PP1 $\alpha$ (Fig. 3B). Thus, Src mediates PP1 $\alpha$ inhibition leading to CaMKII activation. Altogether, we suggest that PP1 is activated by PKC leading to CaMKII inhibition, and inhibition of PP1 by Src leads to CaMKII activation. The possible involvement of Src in PP2A inhibition in mouse sperm has been suggested elsewhere (Krapf et al. 2010). Furthermore, we show in bovine sperm that Src mediates PKA-dependent EGFR phosphorylation on Tyr-845, a known Src-specific phosphorylation site (Etkovitz et al. 2009). We also show that Src mediates Tyr-438 phosphorylation/ inactivation of the actin-severing protein, gelsolin, leading to an increase in actin polymerization and hyper-activated motility during human sperm capacitation (Finkelstein et al. 2013). In our previous study,

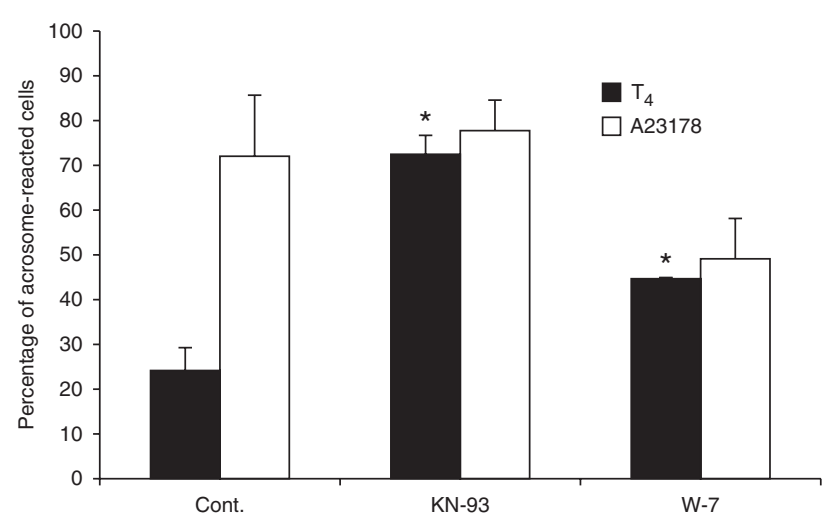

Figure 7 CaMKII enhances the spontaneous acrosome reaction. Bovine spermatozoa were incubated in capacitation medium for $4 \mathrm{~h}$ with or without KN-93 $(50 \mu \mathrm{M})$ or W-7 $(50 \mu \mathrm{M})$. Calcium ionophore (A23187, $10 \mu \mathrm{M}$ ) was added for an additional $20 \mathrm{~min}$. Sperm samples were smeared on slides to determine acrosome reaction, as described in the 'materials and methods' section. These data represent the mean \pm s.D. of duplicates from at least three experiments. * Significant difference from the corresponding control, $P<0.05$. 


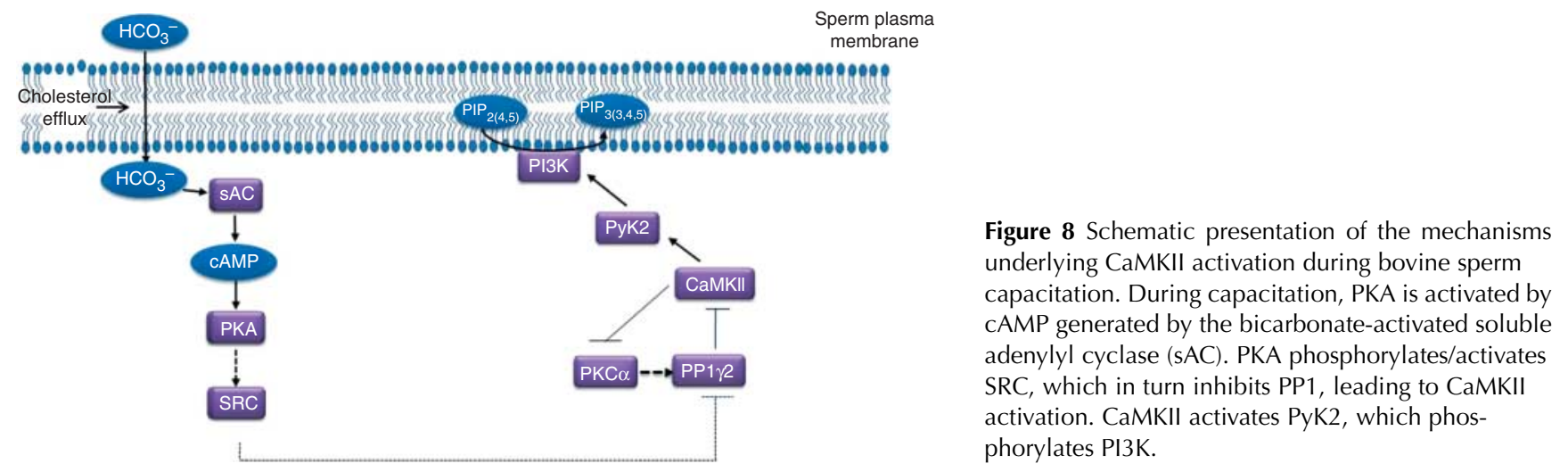

we suggested that Src can be activated by PKA in bovine sperm (Etkovitz et al. 2009). This conclusion was further supported by others in human sperm (Mitchell et al. 2008). Here, we show that inhibition of PKA caused significant inhibition of CaMKII phosphorylation and activating PKA by adding 8Br-cAMP to the cells stimulated CaMKII phosphorylation (Fig. 4A and B). Furthermore, the enhanced effect of 8Br-cAMP on PI3K phosphorylation was inhibited by KN-93 (Fig. 4C). These data suggest that PKA mediates CaMKII activation through the following mechanism: PKA activates Src that inhibit PP1 $\alpha$ leading to CaMKII activation as suggested earlier. In a recent study, we suggested that PKA can mediate PKC $\alpha$ dephosphorylation/inactivation; however, PP1 is not responsible for direct PKC dephosphorylation, as inhibition of PP1 did not influence the PKC phosphorylation level (Rotman et al. 2010). In this study, we suggest a mechanism for this effect, in which PKA activates Src, which mediates PP1 $\alpha$ inhibition, leading to CaMKII activation and PKC inhibition. It was shown elsewhere that PKA can directly phosphorylate/ activate CaMK (Ferrero et al. 2007); however, in this study, we showed that CaMKII phosphorylation is completely inhibited by inhibition of Src (Fig. 3B); thus, we conclude that Src mediates CaMKII phosphorylation in bovine sperm.

Next, we tried to understand the relationships between CaMKII and PI3K. We show that PI3K tyrosine phosphorylation during sperm capacitation is inhibited by KN-93 (Fig. 5A), indicating that CaMK mediates this phosphorylation. Inhibition of PKC by GF or its downregulation by long incubation with PMA or inhibition of PP1 caused a significant increase in p-PI3K, which was inhibited by KN-93 (Fig. 5B and C), further supporting the phosphorylation of PI3K by CaMK. This finding is also supported by the fact that inhibition of PKC or PP1 caused CaMKII activation, as well (Figs 1 and 3A). In order to explain these relationships, we tested the involvement of Pyk2, and we found that inhibition of PP1 enhanced Pyk2 phosphorylation, which is inhibited by KN-93 (Fig. 6), indicating that CaMKII activates Pyk2, a known activator of PI3K. Other studies showed that
CaMKII can activate the tyrosine kinase Pyk2 (Della Rocca et al. 1997, Soltoff 1998, Zwick et al. 1999, Heidinger et al. 2002, Fan et al. 2005, Montiel et al. 2007), which can phosphorylate PI3K on tyrosine (Dikic et al. 1996, Schlaepfer \& Hunter 1996, Avraham et al. 2000, Rocic et al. 2001).

In our previous publication, we showed PKA-dependent activation of PI3K and inhibition by PKC in bovine sperm capacitation (Etkovitz et al. 2007). However, the activation of PI3K by PKA is not due to its possible inhibitory effect on PKC, as inhibition of PKA abrogated the enhanced effect of PKC inhibition on p-PI3K (Rotman et al. 2010). Thus, PKC inhibition by itself cannot activate PI3K unless PKA is active. It is known that PKA can phosphorylate $\mathrm{p} 85$, the regulatory subunit of $\mathrm{PI} 3 \mathrm{~K}$ on Ser-83, which is important for enzyme activation (Cosentino et al. 2007). Here, we further suggest a mechanism by which PKA mediates Tyr-458 phosphorylation of $\mathrm{PI} 3 \mathrm{~K}$. We propose the following cascade: PKA activates Src, which inhibits PP1, leading to CaMK activation. This leads to Pyk2 activation and tyrosine phosphorylation of PI3K. Incubation of the sperm in non-capacitation medium (NKM medium) revealed relatively rapid dephosphorylation of CaMKII (Fig. 2). Under these conditions, PKA cannot be activated as there is no bicarbonate in the medium. Bicarbonate activates SAC that comprises $95 \%$ of sperm AC; thus, in the absence of bicarbonate, cAMP is not practically produced and no PKA activation occurs. According to our model, when PKA is inactive, PP1 is active, resulting in CaMKII dephosphorylation.

It is not clear what function CaMKII fulfills in sperm physiology besides its regulation of $\mathrm{PI} 3 \mathrm{~K}$, which participates in actin polymerization during capacitation (Etkovitz et al. 2007) and in the acrosome reaction (Etkovitz et al. 2009, Breitbart et al. 2010). Here, we showed that inhibition of CaMK caused high increase in spontaneous acrosome reaction (Fig. 7). This may suggest that active CaMKII protects the sperm cell from prematurely undergoing the acrosome reaction. In a recent study, it was suggested that CaMKII interacts with the protein, MUPP1, and prevents spontaneous 
acrosomal exocytosis in mouse sperm (Ackermann et al. 2009). MUPP1 is present in the acrosomal region of sperm of various mammals, including bovine sperm (Heydecke et al. 2006), and mediates the recruitment of molecules that control the initial tethering of acrosomespecific sites to the plasma membrane (Ackermann et al. 2009). It is possible that CaMKII catalyzes the phosphorylation of proteins responsible for keeping the acrosomal vesicles in an intermediate pre-assembled fusion state (Wang 2008), thereby preventing spontaneous acrosome reaction. Although a recent study shows that fertilizing mouse sperm undergoes the acrosome reaction within the cumulus (Jin et al. 2011), it is still accepted that the physiological acrosome reaction occurs after the binding of the capacitated sperm to the egg zona pellucida (Ward \& Kopf 1993). Thus, sperm that undergo a spontaneous acrosome-like reaction before reaching the egg region cannot fertilize the egg. We recently showed that human sperm with a relatively high spontaneous acrosome reaction rate exhibit poor IVF, while low spontaneous acrosome reaction is associated with high fertilization potential (Wiser et al. 2013).

Figure 8 summarizes the pathway suggested by our findings. CaMKII is known to undergo self-phosphory lation and we suggest that this phosphorylation is also maintained by the ability of CaMKII to inhibit PKC/PP1 . Therefore, inhibition of PP1 by calyculin A activates CaMKII and Pyk2, leading to PI3K activation. Moreover, PKA activates Src, which in turn mediates PP1 inhibition leading to CaMKII activation. Our data support our previous conclusion that the ratio between PKA and PKC activity during capacitation regulates PI3K activity (Etkovitz et al. 2007); activation of PKA enhances PI3K activity whereas activation of PKC reduces PI3K activity (Etkovitz et al. 2007). This study sheds light on the mechanism of this regulation, which is now better understood. We suggest that PKA activates PI3K via Src-dependent PP1 inhibition, and PKC inactivates PI3K via activation of PP1. Thus, PP1 plays a key role in this process, as its activity is enhanced by PKC and inhibited via PKA/Src, or CaMKII, which inhibits PKC leading to PP1 inhibition. PP1 activation causes dephosphorylation/ inactivation of CaMKII, while PP1 inhibition maintains CaMKII in a high phosphorylation/activation state, leading to the activation of the tyrosine kinase Pyk2, which phosphorylates PI3K on tyrosine 458.

\section{Declaration of interest}

The authors declare that there is no conflict of interest that could be perceived as prejudicing the impartiality of the research reported.

\section{Funding}

This work was supported by the IHEL Foundation to H Breitbart.

\section{References}

Ackermann F, Zitranski N, Borth H, Buech T, Gudermann T \& Boekhoff I 2009 CaMKIl $\alpha$ interacts with multi-PDZ domain protein MUPP1 in spermatozoa and prevents spontaneous acrosomal exocytosis. Journal of Cell Science 122 4547-4557. (doi:10.1242/jcs.058263)

Almog T, Lazar S, Reiss N, Etkovitz N, Milch E, Rahamim N, DobkinBekman M, Rotem R, Kalina M, Ramon J et al. 2008 Identification of extracellular signal-regulated kinase $1 / 2$ and p38MAPK as regulators of human sperm motility and acrosome reaction and as predictors of poor spermatozoa quality. Journal of Biological Chemistry 283 14479-14489. (doi:10.1074/jbc.M710492200)

Ashizawa K, Wishart GJ, Katayama S, Takano D, Ranasinghe AR, Narumi K \& Tsuzuki Y 2006 Regulation of acrosome reaction of fowl spermatozoa: evidence for the involvement of protein kinase $\mathrm{C}$ and protein phosphatase-type 1 and/or -type 2A. Reproduction 131 1017-1024. (doi:10.1530/rep.1.01069)

Avraham H, Park SY, Schinkmann K \& Avraham S 2000 RAFTK/ Pyk2-mediated cellular signalling. Cellular Signalling 12 123-133. (doi:10.1016/S0898-6568(99)00076-5)

Baker MA, Hetherington L \& Aitken RJ 2006 Identification of SRC as a key PKA-stimulated tyrosine kinase involved in the capacitation-associated hyperactivation of murine spermatozoa. Journal of Cell Science 119 3182-3192. (doi:10.1242/jcs.03055)

Breitbart H 2003 Signaling pathways in sperm capacitation and acrosome reaction. Cellular and Molecular Biology 49 321-327.

Breitbart H \& Naor Z 1999 Protein kinases in mammalian sperm capacitation and the acrosome reaction. Reviews of Reproduction 4 151-159. (doi:10.1530/ror.0.0040151)

Breitbart H, Lax Y, Rotem R \& Naor Z 1992 Role of protein kinase C in the acrosome reaction of mammalian spermatozoa. Biochemical Journal $281473-476$.

Breitbart H, Rotman T, Rubinstein S \& Etkovitz N 2010 Role and regulation of $\mathrm{PI} 3 \mathrm{~K}$ in sperm capacitation and the acrosome reaction. Molecular and Cellular Endocrinology 314 234-238. (doi:10.1016/ j.mce.2009.06.009)

Cantley LC 2002 The phosphoinositide 3-kinase pathway. Science 296 1655-1657. (doi:10.1126/science.296.5573.1655)

Chakrabarti R, Cheng L, Puri P, Soler D \& Vijayaraghavan S 2007 Protein phosphatase PP1 $\gamma 2$ in sperm morphogenesis and epididymal initiation of sperm motility. Asian Journal of Andrology 9 445-452. (doi:10.1111/ j.1745-7262.2007.00307.x)

Cohen G, Rubinstein S, Gur Y \& Breitbart H 2004 Crosstalk between protein kinase $\mathrm{A}$ and $\mathrm{C}$ regulates phospholipase $\mathrm{D}$ and $\mathrm{F}$-actin formation during sperm capacitation. Developmental Biology 267 230-241. (doi:10.1016/j.ydbio.2003.10.034)

Cosentino C, Di Domenico M, Porcellini A, Cuozzo C, De Gregorio G, Santillo MR, Agnese S, Di Stasio R, Feliciello A, Migliaccio A et al. 2007 p85 regulatory subunit of PI3K mediates CAMP-PKA and estrogens biological effects on growth and survival. Oncogene 26 2095-2103. (doi:10.1038/sj.onc.1210027)

De Gregorio G, Coppa A, Cosentino C, Ucci S, Messina S, Nicolussi A, D'Inzeo S, Di Pardo A, Avvedimento EV \& Porcellini A 2007 The p85 regulatory subunit of PI3K mediates TSH-CAMP-PKA growth and survival signals. Oncogene 26 2039-2047. (doi:10.1038/sj.onc. 1210011)

Della Rocca GJ, van Biesen T, Daaka Y, Luttrell DK, Luttrell LM \& Lefkowitz RJ 1997 Ras-dependent mitogen-activated protein kinase activation by $\mathrm{G}$ protein-coupled receptors - convergence of $\mathrm{G}(\mathrm{i})$ - and G(q)-mediated pathways on calcium/calmodulin, Pyk2, and Src kinase. Journal of Biological Chemistry 272 19125-19132. (doi:10.1074/jbc. 272.31.19125)

Dikic I, Tokiwa G, Lev S, Courtneidge SA \& Schlessinger J 1996 A role for Pyk2 and Src in linking G-protein-coupled receptors with MAP kinase activation. Nature 383 547-550. (doi:10.1038/383547a0)

Etkovitz N, Rubinstein S, Daniel L \& Breitbart H 2007 Role of PI3-kinase and PI4-kinase in actin polymerization during bovine sperm capacitation. Biology of Reproduction 77 263-273. (doi:10.1095/biolreprod. 106.056705)

Etkovitz N, Tirosh Y, Chazan R, Jaldety Y, Daniel L, Rubinstein S \& Breitbart H 2009 Bovine sperm acrosome reaction induced by 
G protein-coupled receptor agonists is mediated by epidermal growth factor receptor transactivation. Developmental Biology 334 447-457. (doi:10.1016/j.ydbio.2009.08.002)

Fan RS, Jacamo RO, Jiang XH, Sinnett-Smith J \& Rozengurt E 2005 $\mathrm{G}$ protein-coupled receptor activation rapidly stimulates focal adhesion kinase phosphorylation at Ser-843 - mediation by $\mathrm{Ca}^{2+}$, calmodulin, and $\mathrm{Ca}^{2+}$ calmodulin-dependent kinase II. Journal of Biological Chemistry 280 24212-24220. (doi:10.1074/jbc.M500716200)

Ferrero P, Said M, Sanchez G, Vittone L, Valverde C, Donoso P, Mattiazzi A \& Mundina-Weilenmann C $2007 \mathrm{Ca}^{2+}$ /calmodulin kinase II increases ryanodine binding and $\mathrm{Ca}^{2+}$-induced sarcoplasmic reticulum $\mathrm{Ca}^{2+}$ release kinetics during $\beta$-adrenergic stimulation. Journal of Molecular and Cellular Cardiology 43 281-291. (doi:10.1016/j.yjmcc.2007. 05.022)

Finkelstein M, Megnagi B, Ickowicz D \& Breitbart H 2013 Regulation of sperm motility by PIP2 $(4,5)$ and actin polymerization. Developmental Biology 381 62-72. (doi:10.1016/j.ydbio.2013.06.014)

Fisher HM, Brewis IA, Barratt CL, Cooke ID \& Moore HD 1998 Phosphoinositide 3-kinase is involved in the induction of the human sperm acrosome reaction downstream of tyrosine phosphorylation. Molecular Human Reproduction 4 849-855. (doi:10.1093/ molehr/4.9.849)

Furuya S, Endo Y, Osumi K, Oba M, Nozawa S \& Suzuki S 1993 Calyculin A, protein phosphatase inhibitor, enhances capacitation of human sperm. Fertility and Sterility 59 216-222.

González-Fernández L, Macías-García B, Loux SC, Varner DD \& Hinrichs K 2013 Focal adhesion kinases and calcium/calmodulindependent protein kinases regulate protein tyrosine phosphorylation in stallion sperm. Biology of Reproduction 88 138. (doi:10.1095/biolreprod.112.107078)

Greengard P, Valtorta F, Czernik AJ \& Benfenati F 1993 Synaptic vesicle phosphoproteins and regulation of synaptic function. Science 259 780-785. (doi:10.1126/science.8430330)

Guan L, Song K, Pysz MA, Curry KJ, Hizli AA, Danielpour D, Black AR \& Black JD 2007 Protein kinase C-mediated down-regulation of cyclin D1 involves activation of the translational repressor 4E-BP1 via a phosphoinositide 3-kinase/Akt-independent, protein phosphatase 2Adependent mechanism in intestinal epithelial cells. Journal of Biological Chemistry 282 14213-14225. (doi:10.1074/jbc.M610513200)

Heidinger V, Manzerra P, Wang XQ, Strasser U, Yu SP, Choi DW \& Behrens MM 2002 Metabotropic glutamate receptor 1-induced upregulation of NMDA receptor current: mediation through the Pyk2/Src-family kinase pathway in cortical neurons. Journal of Neuroscience 22 5452-5461.

Heydecke D, Meyer D, Ackermann F, Wilhelm B, Gudermann T \& Boekhoff I 2006 The multi PDZ domain protein MUPP1 as a putative scaffolding protein for organizing signaling complexes in the acrosome of mammalian spermatozoa. Journal of Andrology 27 390-404. (doi:10.2164/jandrol.05166)

Hofmann J 2004 Protein kinase C isozymes as potential targets for anticancer therapy. Current Cancer Drug Targets 4 125-146. (doi:10.2174/1568009043481579)

Huang Z \& Vijayaraghavan S 2004 Increased phosphorylation of a distinct subcellular pool of protein phosphatase, PP1 $\gamma 2$, during epididymal sperm maturation. Biology of Reproduction 70 439-447. (doi:10.1095/ biolreprod.103.020024)

Hudmon A \& Schulman H 2002 Structure-function of the multifunctional $\mathrm{Ca}^{2+} /$ calmodulin-dependent protein kinase II. Biochemical Journal 364 593-611. (doi:10.1042/BJ20020228)

Hunter T \& Schulman H 2005 CaMKII structure - an elegant design. Cell 123 765-767. (doi:10.1016/j.cell.2005.11.017)

Hwang J, Bragado MJ, Duan RD \& Williams JA 1996 Protein phosphatase inhibitors potentiate $\mathrm{Ca}^{2+} /$ calmodulin-dependent protein kinase II activity in rat pancreatic acinar cells. Biochemical and Biophysical Research Communications 225 520-524. (doi:10.1006/ bbrc.1996.1205)

Ignotz GG \& Suarez SS 2005 Calcium/calmodulin and calmodulin kinase II stimulate hyperactivation in demembranated bovine sperm. Biology of Reproduction 73 519-526. (doi:10.1095/biolreprod.105.040733)

Jin M, Fujiwara E, Kakiuchi Y, Okabe M, Satouh Y, Baba SA, Chiba K \& Hirohashi N 2011 Most fertilizing mouse spermatozoa begin their acrosome reaction before contact with the zona pellucida during in vitro fertilization. PNAS 108 4892-4896. (doi:10.1073/pnas.10182 02108)

Jungnickel MK, Sutton KA, Wang Y \& Florman HM 2007 Phosphoinositidedependent pathways in mouse sperm are regulated by egg ZP3 and drive the acrosome reaction. Developmental Biology 304 116-126. (doi:10.1016/j.ydbio.2006.12.023)

Krapf D, Arcelay E, Wertheimer EV, Sanjay A, Pilder SH, Salicioni AM \& Visconti PE 2010 Inhibition of Ser/Thr phosphatases induces capacitation-associated signaling in the presence of Src kinase inhibitors. Journal of Biological Chemistry 285 7977-7985. (doi:10.1074/jbc. M109.085845)

Leal-Ortiz S, Waites CL, Terry-Lorenzo R, Zamorano P, Gundelfinger ED \& Garner CC 2008 Piccolo modulation of Synapsin1a dynamics regulates synaptic vesicle exocytosis. Journal of Cell Biology 181 831-846. (doi:10.1083/jcb.200711167)

Lisman JE \& Zhabotinsky AM 2001 A model of synaptic memory: a CaMKII/PP1 switch that potentiates transmission by organizing an AMPA receptor anchoring assembly. Neuron 31 191-201. (doi:10.1016/ S0896-6273(01)00364-6)

Mallozzi C, De Franceschi L, Brugnara C \& Di Stasi AM 2005 Protein phosphatase $1 \alpha$ is tyrosine-phosphorylated and inactivated by peroxynitrite in erythrocytes through the src family kinase fgr. Free Radical Biology \& Medicine 38 1625-1636. (doi:10.1016/j.freeradbiomed. 2005.02.021)

Mao LM, Yang L, Arora A, Choe ES, Zhang GC, Liu ZG, Fibuch EE \& Wang JQ 2005 Role of protein phosphatase 2A in mGluR5-regulated MEK/ERK phosphorylation in neurons. Journal of Biological Chemistry 280 12602-12610. (doi:10.1074/jbc.M411709200)

Mitchell LA, Nixon B, Baker MA \& Aitken RJ 2008 Investigation of the role of SRC in capacitation-associated tyrosine phosphorylation of human spermatozoa. Molecular Human Reproduction 14 235-243. (doi:10.1093/molehr/gan007)

Montiel M, Quesada J \& Jimenez E 2007 Activation of calcium-dependent kinases and epidermal growth factor receptor regulate muscarinic acetylcholine receptor-mediated MAPK/ERK activation in thyroid epithelial cells. Cellular Signalling 19 2138-2146. (doi:10.1016/ j.cellsig.2007.06.010)

Mwimbi X, Muimo R, Green MW \& Mehta A 2003 Making human nasal cilia beat in the cold: a real time assay for cell signalling. Cellular Signalling 15 395-402. (doi:10.1016/S0898-6568(02)00143-2)

Nomura M, Yoshida M \& Morisawa M 2004 Calmodulin/calmodulindependent protein kinase II mediates SAAF-induced motility activation of ascidian sperm. Cell Motility and the Cytoskeleton 59 28-37. (doi:10.1002/cm.20020)

Parrish JJ, Susko-Parrish J \& Graham JK 1999 In vitro capacitation of bovine spermatozoa: role of intracellular calcium. Theriogenology 51 461-472. (doi:10.1016/S0093-691X(98)00240-4)

Rocic P, Govindarajan G, Sabri A \& Lucchesi PA 2001 A role for PYK2 in regulation of ERK1/2 MAP kinases and PI 3-kinase by ANG II in vascular smooth muscle. American Journal of Physiology. Cell Physiology $\mathbf{2 8 0}$ C90-C99.

Rotman T, Etkovitz N, Spiegel A, Rubinstein S \& Breitbart H 2010 Protein kinase $\mathrm{A}$ and protein kinase $\mathrm{C}(\boldsymbol{\alpha}) / \mathrm{PPP} 1 \mathrm{CC} 2$ play opposing roles in the regulation of phosphatidylinositol 3-kinase activation in bovine sperm. Reproduction 140 43-56. (doi:10.1530/REP-09-0314)

Schlaepfer DD \& Hunter T 1996 Evidence for in vivo phosphorylation of the grb2 SP2-domain binding site on focal adhesion kinase by Src-family protein-tyrosine kinase. Molecular and Cellular Biology $\mathbf{1 6}$ 7182-7184.

Sipeki S, Bander E, Parker PJ \& Farago A 2006 PKC $\alpha$ reduces the lipid kinase activity of the $\mathrm{p} 110 \alpha / \mathrm{p} 85 \alpha \mathrm{PI} 3 \mathrm{~K}$ through the phosphorylation of the catalytic subunit. Biochemical and Biophysical Research Communications 339 122-125. (doi:10.1016/j.bbrc.2005.10.194)

Smith EF 2002 Regulation of flagellar dynein by calcium and a role for an axonemal calmodulin and calmodulin-dependent kinase. Molecular Biology of the Cell 13 3303-3313. (doi:10.1091/mbc.E0204-0185)

Smith PK, Krohn RI, Hermanson GT, Mallia AK, Gartner FH, Provenzano MD, Fujimoto EK, Goeke NM, Olson BJ \& Klenk DC 1985 Measurement of protein using bicinchoninic acid. Analytical Biochemistry 150 76-85. (doi:10.1016/0003-2697(85)90442-7) 
Soltoff SP 1998 Related adhesion focal tyrosine kinase and the epidermal growth factor receptor mediate the stimulation of mitogen-activated protein kinase by the G-protein-coupled P-2Y2 receptor. Journal of Biological Chemistry 273 23110-23117. (doi:10.1074/jbc.273.36. 23110)

Srinivasan M \& Begum N 1994 Stimulation of protein phosphatase-1 activity by phorbol esters. Evaluation of the regulatory role of protein kinase $C$ in insulin action. Journal of Biological Chemistry 269 16662-16667.

Villa-Moruzzi E \& Puntoni F 1996 Phosphorylation of phosphatase-1 $\alpha$ in cells expressing v-src. Biochemical and Biophysical Research Communications 219 863-867. (doi:10.1006/bbrc.1996.0337)

Wang Z-W 2008 Regulation of synaptic transmission by presynaptic CaMKII and BK channels. Molecular Neurobiology 38 153-166. (doi:10.1007/s12035-008-8039-7)
Ward CR \& Kopf GS 1993 Molecular events mediating sperm activation. Developmental Biology 158 1-26. (doi:10.1006/dbio.1993.1165)

Wiser A, Sachar S, Ghetler Y, Shulman A \& Breitbart H 2013 Assessment of sperm hyperactivated motility and acrosome reaction can discriminate the use of spermatozoa for conventional in vitro fertilisation or intracytoplasmic sperm injection: preliminary results. Andrologia .

Zwick E, Wallasch C, Daub H \& Ullrich A 1999 Distinct calciumdependent pathways of epidermal growth factor receptor transactivation and PYK2 tyrosine phosphorylation in PC12 cells. Journal of Biological Chemistry 274 20989-20996. (doi:10.1074/jbc.274.30.20989)

Received 3 November 2013

First decision 4 December 2013

Accepted 7 January 2014 